\title{
Interest in genetic testing among affected men from hereditary prostate cancer families and their unaffected male relatives
}

\author{
Julie N. Harris, PhD, MPH $H^{1}$, Deborah J. Bowen, PhD', Alan Kuniyuki, MS ${ }^{3}$, Laura McIntosh, PhD ${ }^{4}$, \\ Liesel M. FitzGerald, $P h D^{4}$, Elaine A. Ostrander, $P h D^{5}$, and Janet L. Stanford, PhD ${ }^{4,6}$
}

\begin{abstract}
Purpose: The objective of this study was to evaluate potential sociodemographic, medical, psychosocial, and behavioral correlates of interest in genetic testing in men from hereditary prostate cancer families. Methods: Family members affected with prostate cancer $(n=559)$ and their unaffected male relatives $(n=370)$ completed a mailed survey. Multivariable logistic regression models were used to examine the association between potential correlates and interest in genetic testing for prostate cancer. Results: Forty-five percent of affected and 56\% of unaffected men reported that they definitely would take a genetic test for prostate cancer. More affected men reported high levels of familiarity with genetic testing than unaffected men (46 vs. $25 \%$ ). There were several variables that were significantly correlated with interest in either affected or unaffected men, but only age and familiarity with genetics were significant in both groups. After controlling for confounding variables, only familiarity remained a significant correlate in both groups. Conclusions: The contrast between low levels of familiarity with genetics and high test interest among unaffected men highlights the need for increased educational efforts targeting hereditary prostate cancer families. Overall, results illuminated several novel characteristics of men from hereditary prostate cancer families that should be considered when developing future informed consent procedures or educational materials for prostate cancer genetic testing. Genet Med 2009:11(5):344-355.
\end{abstract}

Key Words: prostate cancer, family, hereditary, genetic test, psychosocial

t is estimated that one in six men in the U.S. will be diagnosed with prostate cancer during his lifetime, ${ }^{1}$ and a family history of prostate cancer in a first-degree relative increases a man's risk by 2- to 3-fold. 2,3 Evidence for an hereditary form of prostate cancer has been generated from segregation analyses supporting both autosomal dominant and recessive modes of inheritance of rare, high-risk alleles. In addition, numerous putative loci for hereditary prostate cancer (HPC) have been mapped. ${ }^{4}$ However, specific genes and disease-associated mutations in most of these HPC loci

From the ${ }^{1}$ Robert Wood Johnson Foundation Health and Society Scholars Program at the University of California, Berkeley and San Francisco, California; ${ }^{2}$ Department of Social and Behavioral Sciences, Boston University, Boston, Massachusetts; ${ }^{3}$ Program in Cancer Prevention; ${ }^{4}$ Program in Epidemiology, Fred Hutchinson Cancer Research Center, Seattle, Washington; ${ }^{5}$ Cancer Genetics Branch, National Human Genome Research Institute/ National Institutes of Health, Bethesda, Maryland; and ${ }^{6}$ Epidemiology Department, University of Washington, Seattle, Washington.

Julie N. Harris, PhD, MPH, Robert Wood Johnson Foundation Health and Society Scholar at University of California at San Francisco, 3333 California Street, San Francisco, CA 94118. E-mail: harrisjn@chc.ucsf.edu.

Disclosure: The authors declare no conflict of interest.

Submitted for publication September 18, 2008.

Accepted for publication January 5, 2009.

DOI: $10.1097 /$ GIM.0b013e31819b2425 remain elusive. Recently, genetic linkage analyses followed by association studies identified chromosomal regions on $8 \mathrm{q} 24$, $17 \mathrm{q} 12$, and $17 \mathrm{q} 24$ that are associated with increased risk of prostate cancer. ${ }^{5-7}$ These associations have now been confirmed in numerous studies, leading one group to combine these genetic loci ${ }^{8}$ into a new genetic test based on five SNP genotypes, the Focus $5^{\mathrm{TM}}$ Prostate Cancer Risk Test (www. proactivegenomics.com), to assess prostate cancer risk. DeCode Genetics has also developed a similar test based on eight SNP genotypes (http://www.decodeme. com/information/trait/PCA). Thus, it seems that a genetic test for prostate cancer is imminent, although it remains unclear how useful such a test will be for evaluating risk for sporadic or HPC.

Men from HPC families have about a 5-fold or greater increase in risk for the disease and prostate cancer tends to be diagnosed 6-7 years earlier than in sporadic prostate cancer cases. ${ }^{9}, 10$ A high-risk HPC family is defined as one in which prostate cancer is present in (a) three or more affected firstdegree relatives; or (b) affected men from three successive generations; and/or (c) two first-degree relatives diagnosed before age $55 .{ }^{11}$ Developing a genetic test for prostate cancer would be a promising first step toward reducing disease severity by identifying individuals from these high-risk families who may benefit most from early detection and perhaps cancer prevention interventions. ${ }^{12}$ However, a genetic test is only useful if people are willing to receive testing, to communicate about it with family members, and to act on any early detection or preventive options available. Furthermore, the genetic tests being developed for prostate cancer are likely to necessitate the synthesis of multiple genetic risk factors and other risk information to provide appropriate risk counseling to a patient (as evidenced by the multi-SNP panels developed by deCode, and proactive genomics). This will require more intensive educational efforts on the part of cancer genetics providers to disseminate and interpret increasingly uncertain risk information. Thus, it is important to understand the characteristics of populations interested in genetic testing and attitudes toward and interest in genetic testing before these tests being widely used. Because men with a familial history of prostate cancer may be most likely to be offered a genetic test when one becomes available, understanding their attitudes and intentions toward genetic testing is of particular interest.

Few studies have been conducted on interest in receiving a genetic test for a predisposition to prostate cancer, though there have been many studies on predictors of interest in genetic testing for other types of hereditary cancers (mainly breast cancer). ${ }^{13-20}$ Reported levels of interest in genetic testing for other hereditary cancers are usually high, though rates have been noted to differ based on the type of disease, the presence of a family history of disease, and gender with men usually reporting lower rates of interest and uptake in testing for certain cancers. ${ }^{21}$ 
Of the few investigations of prostate cancer genetic testing, most have primarily sampled men from the general population. ${ }^{22-25}$ A few studies sampled men with a family history of prostate cancer, ${ }^{26-28}$ and only two examined men from HPC families. ${ }^{29,30}$ Both of these studies in HPC families were conducted with unaffected relatives of whom $87-94 \%$ expressed an interest in prostate cancer susceptibility testing.

Furthermore, there was only a single study that analyzed intentions and attitudes regarding prostate cancer heritability in affected men and their unaffected siblings. ${ }^{26}$ The affected men were not queried about their interest in prostate cancer genetic testing. ${ }^{26}$ Thus, we currently know little about how affected men would react to the availability of such a test, nor have the emotional aspects of testing been explored in affected men.

The purpose of this study was to evaluate sociodemographic, psychosocial, medical, and health-related behavioral characteristics with interest in genetic testing for prostate cancer risk in affected and unaffected male relatives from a large collection of HPC families. A secondary objective was to examine differences in the association between risk perceptions and cancer worry and interest in genetic testing among unaffected men in these families.

\section{MATERIALS AND METHODS}

\section{Study population}

Data for these analyses are from the ongoing Prostate Cancer Genetic Research Study (PROGRESS). PROGRESS is a longitudinal study of HPC families that aims to identify genetic loci that are associated with prostate cancer risk. Recruitment commenced in 1995 and families in the current analyses were ascertained through 2000. Once identified, families were included in the study if they had either three or more affected men with prostate cancer, three generations of men affected by prostate cancer, or two first-degree relatives diagnosed with prostate cancer before age $65 .{ }^{31}$

Affected men and their unaffected relatives aged 40 and older were asked to complete a self-administered baseline survey that included questions on family and medical history, prostate cancer screening, health-related behaviors, and sociodemographic variables. A total of 797 affected men and 560 unaffected men completed the baseline survey. A follow-up survey was sent to all participants still alive and enrolled in the study ( $n=677$ affected; $n=496$ unaffected) at about 6 years after baseline (range 3-9 years). The follow-up survey was similar to the baseline survey, with additional questions added about cancer risk perceptions and worry and prostate cancer genetic testing interest and familiarity. A total of $587(86 \%)$ affected men and 378 (76\%) unaffected men completed the follow-up survey.

Only affected and unaffected men who completed both a baseline and follow-up survey and who answered the question regarding interest in genetic testing were included in these analyses. Less than $5 \%$ of men (28 affected and 8 unaffected) were excluded because of missing data for the question on genetic testing. Thus, 257 families with a total of 559 men diagnosed with prostate cancer and 370 male relatives without a diagnosis of prostate cancer were included.

\section{Analysis variables}

\section{Interest in genetic testing}

The main outcome variable was interest in genetic testing for prostate cancer, assessed by a single question in the follow-up survey, "If there were a genetic test for prostate cancer, would you be interested in taking such a test?." There were four response categories: definitely not interested, probably not interested, probably interested, and definitely interested. For these analyses, responses were dichotomized into those "definitely interested" in genetic testing versus all other responses combined.

\section{Sociodemographic and familiarity with genetic testing variables}

Several sociodemographic, screening and health-related behaviors, and psychosocial characteristics were analyzed, including age and racial group as potential correlates of interest in genetic testing. Age was calculated at the time of the follow-up survey as interest in genetic testing was assessed at that time. Baseline assessments of level of education, race, and marital status were included because these variables were not collected in the follow-up survey. We included a single item asking participants to rate "how much they have read or heard about genetic testing for cancer risk" using a four-point Likert scale ranging from almost nothing to a lot.

\section{Health-related behavior variables}

Smoking, alcohol consumption, body mass index, and prostate-specific antigen (PSA) screening were included in the analyses. Smoking was measured at follow-up and classified as never smoked, past smoker, or current smoker. Alcohol consumption was measured at follow-up by a single question regarding the amount of alcohol consumed in the past 6 months. Body mass index was calculated from participant self-report assessed at baseline, dividing weight (in kilograms) by height (in meters) squared. Data on serum PSA screening was measured at follow-up by a single question asking participants to report whether or not they had a PSA test in the past 5 years.

\section{Medical history variables}

Medical history variables including the presence of other hereditary health disorders, other cancer diagnoses, and selfrated health were included in the analyses for both affected and unaffected men. The perception of other hereditary health disorders was measured by participant self-report using a single question, "Do any other types of health problems, disorders, or diseases tend to run in your family (i.e., two or more relatives)?". It is important to note that other hereditary health disorders are a measure of an individual's perception of the burden of hereditary health disorders in their family. Individuals may be aware that a certain disorder occurs frequently in their family, though the disorder may not be recognized as having a strong genetic component. Other nonprostate cancer diagnoses were queried (specific questions included 13 specific cancers with another category for rare sites), and self-rated health was measured by asking participants to rate their level of general health using a five-point Likert scale ranging from excellent to poor.

\section{Prostate cancer variables (affected men only)}

Several variables specific to men with a prostate cancer history were included in the analyses, including age at diagnosis, time since diagnosis, prostate cancer recurrence, prostate cancer stage at diagnosis, and prostate cancer aggressiveness. Age at diagnosis was collected at baseline, whereas time since diagnosis was computed as the number of years from diagnosis to the follow-up survey assessment. Prostate cancer recurrence was measured by participant response to a single item in the follow-up survey. Prostate cancer stage was obtained from 
review of medical records, including surgical and pathology reports, coded according to SEER guidelines and categorized as local, regional, or distant. Disease aggressiveness was categorized as clinically insignificant, moderate, or aggressive based on an algorithm that used prostate cancer stage at diagnosis, Gleason score, diagnostic PSA level, and if applicable, death from metastatic prostate cancer.

\section{Psychosocial characteristics: Risk perception and worry variables}

Cancer worry was measured using a four-item scale modified from Lerman's cancer worry scale. ${ }^{32}$ This modification included a substitution by asking of one's worry about prostate cancer, rather than worry about cancer in general. Item responses used a four-point Likert scale that ranged from "not at all or rarely" to "almost all the time." Standardized (centered and scaled) responses from these four items were summed to form an overall cancer worry score (Cronbach's alpha $=0.69$ ). Cancer worry scores were then used to classify respondents as having little/no worry versus having occasional worry. Cancer risk perceptions were measured by combining three items that have been adapted from previous research. ${ }^{33}$ Participants were asked to rate (a) how likely it is that the average man will develop prostate cancer; (b) how likely it is that you will develop prostate cancer; and (c) overall, rating of your own risk for developing prostate cancer. Standardized responses from the three items were summed to create an overall risk perception score (Cronbach's alpha $=0.72$ ). These scores were subsequently dichotomized to represent either low or high perceived personal risk.

\section{Statistical analysis}

Interest in genetic testing for prostate cancer was examined separately for affected and unaffected men. This main outcome variable was investigated for possible associations with sociodemographic characteristics, familiarity with genetic testing, health-related behaviors, medical history characteristics, and family history of prostate cancer (number of affected family members). Possible associations with variables specific to prostate cancer history (affected men only), level of worry about prostate cancer (unaffected men only), and perceptions about the risk of getting prostate cancer (unaffected men only) were also investigated. Descriptive statistics are presented for all potential correlates. Percentages on the interest in taking a genetic test for prostate cancer are also reported, and are stratified by the levels of each potential correlate examined within a group.

Multivariable logistic regression was used to evaluate possible associations with the various sociodemographic, psychosocial, medical, and health-related behavioral characteristics, modeling the probability of definitely being interested in taking a genetic test for prostate cancer. Separate mixed effects regression models were examined for affected and unaffected men. Because multiple family members were enrolled into the study, random intercepts that correspond to HPC family units were included to adjust for the expected intrafamilial correlation of observations. Because family history is a family-level variable that is associated with family of origin, the number of affected members within an HPC family was also included in all models as an adjustment factor to aid in the removal of family-level variance and to focus on individual-level associations with interest in genetic testing.

All potential sociodemographic, psychosocial, medical, and health-related behavioral characteristics were initially analyzed using models that included a single potential correlate as a predictor variable, along with adjustments for family history and for intrafamilial correlation (these single-predictor models are subsequently referred to as adjusted for family-level variance only). Odds ratios, $95 \%$ confidence intervals, and $P$ values are presented to describe the association between potential correlates and interest in genetic testing for each of the models. Correlates that were shown to have a significant association with the main outcome variable $(P<0.05)$ were thus identified, and subsequently entered simultaneously into a common logistic regression model to determine their individual significance, above and beyond the other correlates. Odds ratios, 95\% confidence intervals, and $P$ value estimates are presented from these adjusted models. Data analysis was performed using SAS Version 9.1.3 (SAS Institute Inc., Cary, NC).

\section{RESULTS}

\section{Characteristics of the population and frequency of interest in genetic testing}

There were 559 men diagnosed with prostate cancer and 370 unaffected male relatives included in the analyses. There were no differences in age, race, education, or marital status between affected men who were included or excluded from the analyses. This was also the case for unaffected men, with the exception of age. Unaffected, excluded men were older compared with unaffected men who were included in the analyses (72.2 and 57.9, respectively, $P=0.0003$ ). As shown in Table 1 , a larger percentage of unaffected men were definitely interested in genetic testing when compared with affected men (56 vs. $45 \%$ ). Because families were collected as part of a study of HPC, the number of family members diagnosed with prostate cancer was high with $\sim 40 \%$ of men reporting six or more affected relatives (first-, second-, and third-degree relatives were included in this assessment of affected relatives).

The group of affected men was older than their unaffected male relatives (current mean age 73 vs. 58 years), which is likely due to the fact that prostate cancer tends to be diagnosed at older ages. ${ }^{34}$ The mean age at diagnosis in our sample was 63.6 years. At the time of follow-up, the mean time since diagnosis was 9.7 years. There seemed to be a general pattern that as age at diagnosis, current age, or age since diagnosis increased, level of interest in genetic testing decreased. For example, $46 \%$ of unaffected men under the age of 65 were definitely interested in genetic testing compared with $23 \%$ over the age of 75. Furthermore, a higher percentage of affected men under the age of 65 were interested in genetic testing when compared with unaffected men over the age of 65 years (65 vs. $46 \%)$.

The majority of men were white, representing $90 \%$ of affected and $94 \%$ of unaffected. The sample had a fairly high level of education, with $47-55 \%$ having a college degree. In the unaffected group, those with higher levels of education were more interested in genetic testing than those with lower levels of education (57\% of graduate/professional school vs. $31 \%$ of those with high school or less). This pattern was somewhat similar in affected men though smaller percentages of those with a graduate/professional school education reported high interest in genetic testing when compared with those with a college degree (44 vs. 54\%). Most of the men in the sample were married or partnered, and about $40 \%$ reported other hereditary disorders. It is important to note that participants' reports of other hereditary health disorders are based on their own perceptions of hereditary diseases in their family. The 
Table 1 Interest in genetic testing for prostate cancer, by participant characteristics in affected and unaffected men from HPC families ${ }^{a}$

\begin{tabular}{|c|c|c|c|c|c|c|}
\hline \multirow[b]{2}{*}{ Characteristics } & \multicolumn{3}{|c|}{ Affected men } & \multicolumn{3}{|c|}{ Unaffected men } \\
\hline & $n^{b}$ & $\%$ & $\begin{array}{l}\% \text { Interested in } \\
\text { genetic testing }\end{array}$ & $n^{b}$ & $\%$ & $\begin{array}{l}\% \text { Interested in } \\
\text { genetic testing }\end{array}$ \\
\hline \multicolumn{7}{|c|}{ Interest in genetic testing for prostate cancer } \\
\hline Not/less interested & 306 & 55 & 0 & 161 & 44 & 0 \\
\hline Definitely interested & 253 & 45 & 100 & 209 & 56 & 100 \\
\hline \multicolumn{7}{|c|}{ Family history (No. family members affected) } \\
\hline $2-3$ & 120 & 21 & 41 & 56 & 15 & 46 \\
\hline $4-5$ & 214 & 38 & 49 & 157 & 42 & 44 \\
\hline$\geq 6$ & 225 & 40 & 44 & 157 & 42 & 42 \\
\hline Median (range) & $5(2-17)$ & - & - & $5(2-17)$ & - & - \\
\hline \multicolumn{7}{|l|}{ Current age (yr) } \\
\hline$\leq 64$ & 95 & 17 & 65 & 274 & 74 & 46 \\
\hline $65-74$ & 199 & 36 & 48 & 56 & 15 & 45 \\
\hline$\geq 75$ & 265 & 47 & 36 & 40 & 11 & 23 \\
\hline Mean (SD) & $73.3(8.6)$ & - & - & $57.9(11.0)$ & - & - \\
\hline \multicolumn{7}{|l|}{ Age at diagnosis (yr) } \\
\hline$\leq 54$ & 66 & 12 & 64 & - & - & - \\
\hline $55-64$ & 225 & 41 & 48 & - & - & - \\
\hline $65-74$ & 221 & 40 & 39 & - & - & - \\
\hline$\geq 75$ & 38 & 7 & 34 & - & - & - \\
\hline Mean (SD) & $63.6(7.7)$ & - & - & - & - & - \\
\hline \multicolumn{7}{|l|}{ Time since diagnosis $(\mathrm{yr})$} \\
\hline $0-7$ & 141 & 25 & 50 & - & - & - \\
\hline $8-9$ & 148 & 26 & 45 & - & - & - \\
\hline $10-11$ & 138 & 25 & 45 & - & - & - \\
\hline$\geq 12$ & 132 & 24 & 40 & - & - & - \\
\hline Mean (SD) & $9.7(3.8)$ & - & - & - & - & - \\
\hline \multicolumn{7}{|l|}{ Race/ethnicity } \\
\hline Caucasian & 505 & 90 & 48 & 347 & 94 & 44 \\
\hline Other race/ethnicity & 54 & 10 & 20 & 23 & 6 & 43 \\
\hline \multicolumn{7}{|l|}{ Education } \\
\hline Graduate or professional school & 138 & 25 & 44 & 99 & 27 & 57 \\
\hline College degree & 122 & 22 & 54 & 104 & 28 & 43 \\
\hline Vocational/some college & 155 & 28 & 45 & 99 & 27 & 38 \\
\hline High school or less & 143 & 26 & 39 & 67 & 18 & 31 \\
\hline \multicolumn{7}{|l|}{ Marital status } \\
\hline Married/partnered & 500 & 89 & 46 & 324 & 88 & 43 \\
\hline Not married & 59 & 11 & 41 & 46 & 12 & 50 \\
\hline \multicolumn{7}{|c|}{ Other hereditary health disorders (self-reported) } \\
\hline No/don't know & 318 & 57 & 41 & 220 & 60 & 43 \\
\hline Yes & 237 & 43 & 51 & 145 & 40 & $\begin{array}{c}45 \\
\text { (Continued) }\end{array}$ \\
\hline
\end{tabular}


Table 1 Continued

\begin{tabular}{|c|c|c|c|c|c|c|}
\hline \multirow[b]{2}{*}{ Characteristics } & \multicolumn{3}{|c|}{ Affected men } & \multicolumn{3}{|c|}{ Unaffected men } \\
\hline & $n^{b}$ & $\%$ & $\begin{array}{l}\% \text { Interested in } \\
\text { genetic testing }\end{array}$ & $n^{b}$ & $\%$ & $\begin{array}{l}\% \text { Interested in } \\
\text { genetic testing }\end{array}$ \\
\hline \multicolumn{7}{|c|}{ Prostate cancer risk perception (scale score) } \\
\hline Lower personal risk & - & - & - & 193 & 53 & 35 \\
\hline Higher personal risk & - & - & - & 173 & 47 & 53 \\
\hline \multicolumn{7}{|l|}{ Cancer worry (scale score) } \\
\hline No/little worry & - & - & - & 244 & 34 & 39 \\
\hline Occasional worry & - & - & - & 126 & 66 & 53 \\
\hline \multicolumn{7}{|l|}{ Familiarity with genetic testing } \\
\hline Almost nothing/relatively little & 301 & 54 & 36 & 278 & 75 & 39 \\
\hline A fair amount/a lot & 258 & 46 & 57 & 91 & 25 & 57 \\
\hline \multicolumn{7}{|l|}{ Body mass index $\left(\mathrm{kg} / \mathrm{m}^{2}\right)$} \\
\hline $15-<25$ & 195 & 35 & 42 & 108 & 30 & 45 \\
\hline $25-<30$ & 282 & 51 & 46 & 185 & 51 & 40 \\
\hline $30-<60$ & 74 & 13 & 53 & 72 & 20 & 49 \\
\hline \multicolumn{7}{|l|}{ Health status (self-reported) } \\
\hline Excellent & 89 & 16 & 48 & 123 & 33 & 53 \\
\hline Very good & 223 & 40 & 48 & 145 & 39 & 42 \\
\hline Poor/fair/good & 246 & 44 & 42 & 102 & 28 & 34 \\
\hline \multicolumn{7}{|c|}{ Amount of alcohol consumed (past $6 \mathrm{mo}$ ) } \\
\hline$<1$ drink $/$ mo & 230 & 41 & 39 & 142 & 39 & 42 \\
\hline$\leq 3$ drinks/wk & 181 & 32 & 53 & 119 & 32 & 44 \\
\hline$\geq 4$ drinks/wk & 146 & 26 & 46 & 106 & 29 & 45 \\
\hline \multicolumn{7}{|l|}{ Smoking status } \\
\hline Nonsmoker & 223 & 40 & 40 & 164 & 45 & 47 \\
\hline Former smoker & 314 & 56 & 49 & 161 & 44 & 39 \\
\hline Current smoker & 21 & 4 & 52 & 42 & 11 & 48 \\
\hline \multicolumn{7}{|c|}{ Prostate-specific antigen (PSA) tests in past $5 \mathrm{yr}$} \\
\hline No/don't know & 16 & 3 & 31 & 48 & 13 & 27 \\
\hline Yes & 543 & 97 & 46 & 321 & 87 & 46 \\
\hline \multicolumn{7}{|c|}{ Diagnosed with any other type of cancer } \\
\hline No/don’t know & 469 & 85 & 46 & 345 & 93 & 43 \\
\hline Yes & 86 & 15 & 40 & 24 & 7 & 50 \\
\hline \multicolumn{7}{|c|}{ Prostate cancer recurrence/progression } \\
\hline No/don't know & 435 & 80 & 44 & - & - & - \\
\hline Yes & 107 & 20 & 51 & - & - & - \\
\hline \multicolumn{7}{|l|}{ Prostate cancer stage at diagnosis } \\
\hline Local & 376 & 69 & 44 & - & - & - \\
\hline Regional/distant & 167 & 31 & 50 & - & - & - \\
\hline \multicolumn{7}{|l|}{ Prostate cancer aggressiveness } \\
\hline Insignificant/moderate & 271 & 50 & 45 & - & - & - \\
\hline Aggressive disease & 275 & 50 & 46 & - & - & - \\
\hline
\end{tabular}


reported rates of other hereditary health disorders may be higher than actual rates of hereditary health disorders because of differences in patient definitions of what constitutes a hereditary disorder. Greater percentages of affected men with another hereditary disorder in their family reported high interest in genetic testing ( 51 vs. $41 \%$ ).

Among unaffected men, about half (47\%) perceived that they had a higher than average risk for getting prostate cancer. Fifty-three percent of those reporting a high perceived risk indicated that they were definitely interested in prostate cancer genetic testing. This was compared to $35 \%$ of those with a lower perceived risk. Overall, none of the unaffected men reported high levels of worry, but $\sim 66 \%$ reported having occasional worry about prostate cancer in the past month. Fifty-three percent of those with occasional worry reported high levels of interest, compared with $39 \%$ of unaffected men with no or little worry.

Unaffected men reported lower levels of familiarity with genetic testing when compared with affected men ( 75 vs. $54 \%$ ). Those men who were more familiar with genetics reported greater interest when compared with those who were less familiar (57 vs. 36-39\%). More unaffected compared with affected men reported excellent health status (33 vs. 16\%). Of those unaffected men reporting excellent health status, 53\% reported interest in genetic testing compared with $42 \%$ of those in very good health and $34 \%$ of those in poor, fair, or good health.

Ninety-seven percent of affected and $87 \%$ of unaffected men had a PSA test in the past 5 years. In both groups, higher percentages of those who had a PSA test reported interest in genetic testing ( $46 \%$ in both affected and unaffected) than those who had not had a PSA test (31\% affected, $27 \%$ unaffected).

Fifteen percent of affected and 7\% of unaffected men reported being diagnosed with another type of cancer. Among the affected men, $20 \%$ reported that they had had a recurrence or progression of prostate cancer and these individuals were more likely to report interest in genetic testing when compared with those who had not had a recurrence (51 vs. 44\%). Sixty-nine percent of affected men had localized prostate cancer at diagnosis and about $50 \%$ had more aggressive prostate cancer (based on having a Gleason score of $7=4+3$ or $8-10$, or regional/distant stage, or a diagnostic PSA level of $\geq 20 \mathrm{ng} / \mathrm{mL}$ or death from metastatic prostate cancer before age 65). Smaller percentages of those with localized cancer were likely to report interest in genetic testing when compared with those with regional/distant stage disease (44 vs. $50 \%$ ).

\section{Model assessing associations between participant characteristics and interest in genetic testing}

In Table 2, odds ratios, 95\% confidence intervals and corresponding $P$-values are shown for each of the variables, adjusted for family history and correlation within families. In both affected and unaffected men, age and familiarity with genetic testing were significantly associated with interest in prostate cancer genetic testing (omnibus $P<0.05$ ). There was an inverse relationship between age and interest in prostate cancer genetic testing for both groups. For example, affected men aged 65-74 years had an OR of 0.51 (95\% CI: $0.3-0.85)$ for reporting high interest in genetic testing relative to those aged $<65$ years. Unaffected men had a similar pattern to affected men for the stratum aged 75 or older, where both groups were substantially less interested in genetic testing compared with men under age 65 years. Familiarity with genetic testing was also associated with higher levels of interest in genetic testing such that those who reported knowing a fair amount or a lot about prostate cancer genetic testing were more likely to be interested than those who reported knowing almost nothing or relatively little. In affected men, those who reported, "reading or hearing a fair amount" about genetics had 2.36 (95\% CI: 1.67-3.32) times the odds of reporting interest in genetic testing than those who were less familiar with genetics. In unaffected men there was a similar association $(\mathrm{OR}=2.11 ; 95 \% \mathrm{CI}$ : $1.30-1.42)$.

Age at diagnosis, race/ethnicity, and the amount of alcohol consumed in the past 6 months were also associated with interest in genetic testing though only in the affected men. Age at diagnosis had a similar inverse relationship to that seen in age at time of follow-up survey. For example, affected men diagnosed between 55 and 64 years of age had about $0.53(95 \%$ CI:0.30-0.94) times the odds of being interested in receiving genetic testing for prostate cancer than those diagnosed younger than age 55 years. Affected men who belonged to a racial/ethnic minority group were significantly less likely to be highly interested compared with their Caucasian counterparts $(\mathrm{OR}=0.28$; 95\% CI: 0.14-0.57). Finally, affected men who reported another hereditary health disorder in their family had a 1.53 greater odds (95\% CI: $1.08-2.16)$ of reporting that they were highly interested compared with those who reported no other hereditary health disorders.

Education, PSA testing in the past 5 years, and self-reported health were associated with interest in genetic testing in unaffected men but not in affected men. Education had a direct relationship with interest in genetic testing such that those with the highest levels of education were also those most likely to report being highly interested. Designating graduate/professional school as the referent group, the odds of being highly interested in genetic testing among those with some college or vocational schooling were 0.48 (95\% CI: $0.27-0.85)$ times the odds of those who attended graduate/professional school. The odds of being highly interested among those with a high school education or less when compared with graduate/professional school education were 0.35 (95\% CI: $0.18-0.69)$. PSA testing was also associated with interest in that unaffected men who had a PSA test in the past 5 years had 2.34 (95\% CI: 1.18-4.64) times the odds of reporting interest in genetic testing than those who had not had a PSA test. Self-reported health was associated with interest in prostate cancer genetic testing such that those with the lowest levels of health were less likely to report interest. For example, unaffected men reporting poor, fair, or good health had 0.47 (95\% CI: $0.27-0.80)$ times the odds of reporting high levels of interest than those with excellent health. None of the other variables evaluated was significantly related to interest in genetic testing.

\section{Multivariable model assessing associations between participant characteristics and interest in genetic testing}

The variables associated with interest from Table 2 were included in a multivariate adjusted model (Table 3), adjusted for family history, correlation of responses within families, current age, and familiarity with genetic testing for both affected and unaffected men. The model for the affected men also adjusted for age at diagnosis, other hereditary health disorders in the family, and the amount of alcohol consumed in the past 6 months. The model for the unaffected men also adjusted for education, self-reported health status, and PSA testing in the past 5 years

Among affected men, only race/ethnicity and familiarity with genetic testing remained significant predictors of interest in 
Table 2 Odds ratios (OR) and 95\% confidence intervals (CI) for interest in genetic testing for prostate cancer, by affected status in HPC families ${ }^{a, b}$

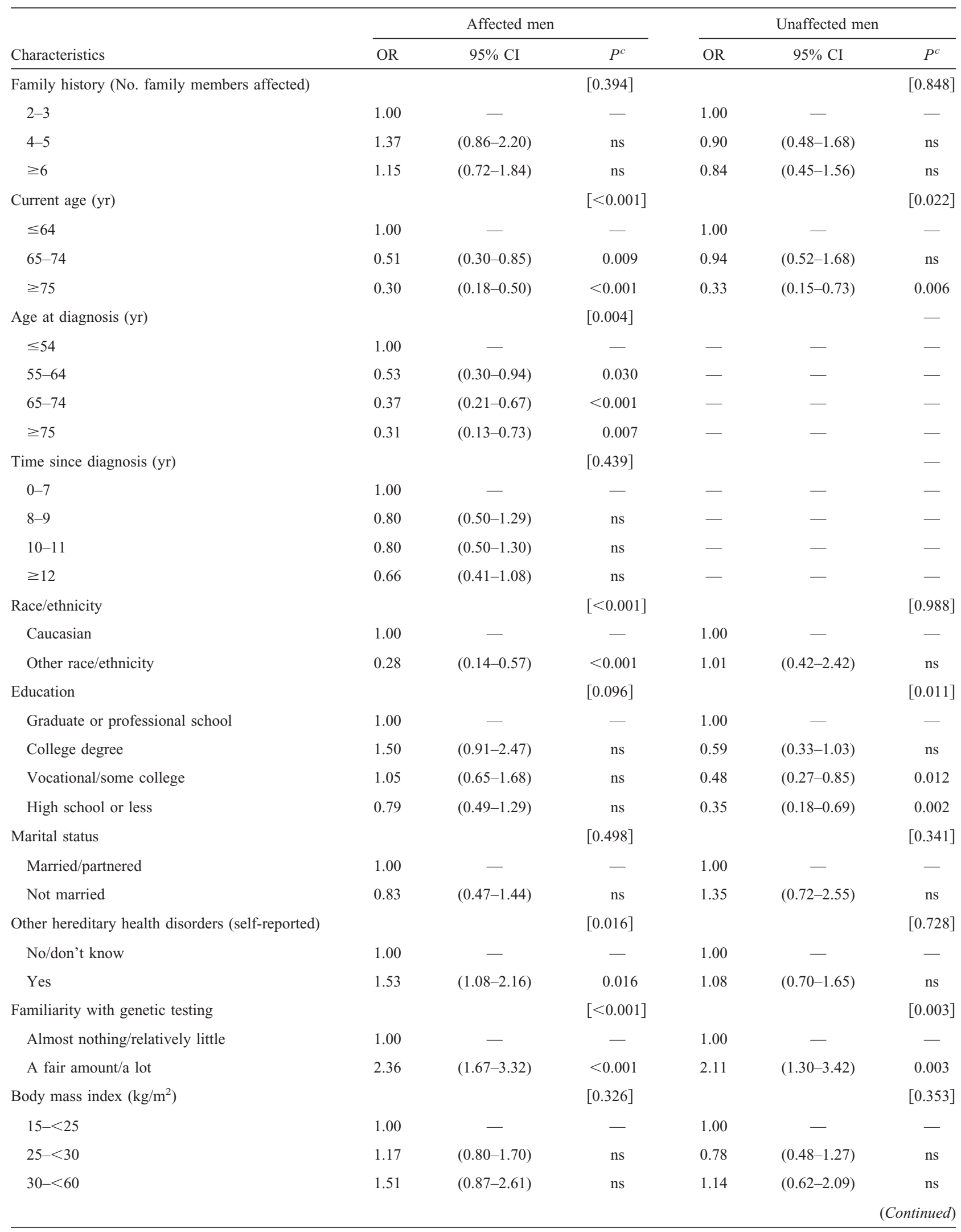


Table 2 Continued

\begin{tabular}{|c|c|c|c|c|c|c|}
\hline \multirow[b]{2}{*}{ Characteristics } & \multicolumn{3}{|c|}{ Affected men } & \multicolumn{3}{|c|}{ Unaffected men } \\
\hline & OR & $95 \% \mathrm{CI}$ & $P^{c}$ & OR & $95 \% \mathrm{CI}$ & $P^{c}$ \\
\hline Health status (self-reported) & & & {$[0.347]$} & & & {$[0.021]$} \\
\hline Excellent & 1.00 & - & - & 1.00 & - & - \\
\hline Very good & 0.92 & $(0.56-1.52)$ & ns & 0.65 & $(0.40-1.06)$ & ns \\
\hline Poor/fair/good & 0.74 & $(0.45-1.21)$ & ns & 0.47 & $(0.27-0.80)$ & 0.006 \\
\hline Amount of alcohol consumed (past $6 \mathrm{mo}$ ) & & & {$[0.025]$} & & & {$[0.842]$} \\
\hline$<1$ drink/mo & 1.00 & - & - & 1.00 & - & - \\
\hline$\leq 3$ drinks/wk & 1.74 & $(1.17-2.60)$ & 0.007 & 1.10 & $(0.66-1.81)$ & ns \\
\hline$\geq 4$ drinks $/$ wk & 1.36 & $(0.88-2.08)$ & ns & 1.16 & $(0.69-1.95)$ & ns \\
\hline Smoking status & & & {$[0.134]$} & & & {$[0.255]$} \\
\hline Nonsmoker & 1.00 & - & - & 1.00 & - & - \\
\hline Former smoker & 1.41 & $(0.99-2.01)$ & ns & 0.70 & $(0.45-1.10)$ & ns \\
\hline Current smoker & 1.66 & $(0.67-4.12)$ & ns & 1.03 & $(0.52-2.05)$ & ns \\
\hline Prostate-specific antigen (PSA) tests in past $5 \mathrm{yr}$ & & & {$[0.308]$} & & & {$[0.015]$} \\
\hline No/don't know & 1.00 & - & - & 1.00 & - & - \\
\hline Yes & 1.75 & $(0.59-5.16)$ & ns & 2.34 & $(1.18-4.64)$ & 0.015 \\
\hline Diagnosed with any other type of cancer & & & {$[0.316]$} & & & {$[0.530]$} \\
\hline No/don't know & 1.00 & - & - & 1.00 & - & - \\
\hline Yes & 0.78 & $(0.49-1.26)$ & ns & 1.31 & $(0.57-3.02)$ & ns \\
\hline Prostate cancer recurrence/progression & & & {$[0.185]$} & & & - \\
\hline No/don't know & 1.00 & - & - & - & - & - \\
\hline Yes & 1.33 & $(0.87-2.05)$ & ns & - & - & - \\
\hline Prostate cancer stage at diagnosis & & & {$[0.217]$} & & & - \\
\hline Local & 1.00 & - & - & - & - & - \\
\hline Regional/distant & 1.26 & $(0.87-1.83)$ & ns & - & - & - \\
\hline Prostate cancer aggressiveness & & & {$[0.728]$} & & & - \\
\hline Insignificant/moderate & 1.00 & - & - & - & - & - \\
\hline Aggressive disease & 1.06 & $(0.76-1.49)$ & ns & - & - & - \\
\hline
\end{tabular}

${ }^{a}$ Based on data from 929 men (257 families): 559 affected men (249 families) and 370 unaffected men (166 families); modeling the probability of being definitely interested in taking a genetic test for prostate cancer.

${ }^{b}$ Logistic regression models include a single characteristic as a predictor, along with adjustments for family history (No. affected family members) and for intrafamilial correlation.

${ }^{c} P$-value for the omnibus test of a predictor is enclosed within brackets.

ns, nonsignificant $(P>0.050)$.

prostate cancer genetic testing when adjusting for other variables (Table 3 ). In the ethnic minority population, the odds of having high levels of interest in genetic testing was 0.25 (95\% CI: 0.12-0.54) times that of Caucasian men. Familiarity with genetic testing also retained a strong association with interest in genetic testing when controlling for the other covariates, such that affected men with a fair amount or a lot of familiarity with genetic testing had 2.34 times the odds of those with less familiarity to report high levels of interest in genetic testing.

Among the unaffected men, current age, familiarity with genetic testing, and PSA testing in the past 5 years remained significant predictors of interest in prostate cancer genetic testing (Table 3). The association between age and interest was somewhat weaker as those in the 65-74 age group did not differ significantly from the younger men. However, unaffected men older than 74 years had $0.31(95 \% \mathrm{CI}$ : $0.13-0.75)$ times the likelihood of reporting high levels of interest in genetic testing when compared with men aged 64 years and under. Similar to affected men, unaffected men reporting a fair amount or a lot of familiarity with genetic testing had 2.16 (95\% CI: 1.28-3.64) times the odds of those with less familiarity to report high levels of interest in genetic testing. Finally, unaffected men who had a PSA test in the last 5 years were 2.22 (95\% CI: $1.08-4.60)$ 
Table 3 Multivariable models: Adjusted odds ratio (OR) and $95 \%$ confidence interval (Cl) estimates by affected status in HPC families, predicting interest in genetic testing for prostate cancer ${ }^{a, b}$

\begin{tabular}{|c|c|c|c|c|c|c|}
\hline \multirow[b]{2}{*}{ Characteristics } & \multicolumn{3}{|c|}{ Affected men } & \multicolumn{3}{|c|}{ Unaffected men } \\
\hline & OR & $95 \% \mathrm{CI}$ & $P^{c}$ & OR & $95 \% \mathrm{CI}$ & $P^{c}$ \\
\hline Family history (No. family members affected) & & & {$[0.551]$} & & & {$[0.797]$} \\
\hline $2-3$ & 1.00 & - & - & 1.00 & - & - \\
\hline $4-5$ & 1.32 & $(0.80-2.20)$ & ns & 0.86 & $(0.44-1.68)$ & ns \\
\hline$\geq 6$ & 1.23 & $(0.74-2.02)$ & ns & 0.79 & $(0.40-1.57)$ & ns \\
\hline Current age (yr) & & & {$[0.074]$} & & & [0.029] \\
\hline$\leq 64$ & 1.00 & - & - & 1.00 & - & - \\
\hline $65-74$ & 0.50 & $(0.23-1.07)$ & $\mathrm{ns}$ & 1.04 & $(0.56-1.95)$ & $\mathrm{ns}$ \\
\hline$\geq 75$ & 0.36 & $(0.15-0.87)$ & 0.023 & 0.31 & $(0.13-0.75)$ & 0.009 \\
\hline Age at diagnosis (yr) & & & [0.994] & & & - \\
\hline$\leq 54$ & 1.00 & - & - & - & - & - \\
\hline $55-64$ & 1.00 & $(0.43-2.31)$ & ns & - & - & - \\
\hline $65-74$ & 1.08 & $(0.40-2.87)$ & ns & - & - & - \\
\hline$\geq 75$ & 1.10 & $(0.32-3.73)$ & ns & - & - & - \\
\hline Race/ethnicity & & & {$[<0.001]$} & & & - \\
\hline Caucasian & 1.00 & - & - & - & - & - \\
\hline Other race/ethnicity & 0.25 & $(0.12-0.54)$ & $<0.001$ & - & - & - \\
\hline Education & & & - & & & [0.139] \\
\hline Graduate or professional school & - & - & - & 1.00 & - & - \\
\hline College degree & - & - & - & 0.65 & $(0.36-1.17)$ & ns \\
\hline Vocational/some college & - & - & - & 0.54 & $(0.29-1.00)$ & 0.049 \\
\hline High school or less & - & - & - & 0.47 & $(0.23-0.99)$ & 0.047 \\
\hline Other hereditary health disorders (self-reported) & & & {$[0.056]$} & & & - \\
\hline No/don't know & 1.00 & - & - & - & - & - \\
\hline Yes & 1.44 & $(0.99-2.10)$ & ns & - & - & - \\
\hline Familiarity with genetic testing & & & {$[<0.001]$} & & & {$[0.004]$} \\
\hline Almost nothing/relatively little & 1.00 & - & - & 1.00 & - & - \\
\hline A fair amount/a lot & 2.34 & $(1.61-3.39)$ & $<0.001$ & 2.16 & $(1.28-3.64)$ & 0.004 \\
\hline Health status (self-reported) & & & - & & & {$[0.521]$} \\
\hline Excellent & - & - & - & 1.00 & - & - \\
\hline Very good & - & - & - & 0.79 & $(0.47-1.32)$ & ns \\
\hline Poor/fair/good & - & - & - & 0.72 & $(0.38-1.35)$ & ns \\
\hline Amount of alcohol consumed (past 6 mo) & & & {$[0.200]$} & & & - \\
\hline$<1 \mathrm{drink} / \mathrm{mo}$ & 1.00 & - & - & - & - & - \\
\hline$\leq 3$ drinks/wk & 1.48 & $(0.95-2.31)$ & ns & - & - & - \\
\hline$\geq 4$ drinks/wk & 1.08 & $(0.68-1.72)$ & ns & - & - & - \\
\hline Prostate-specific antigen (PSA) tests in past $5 \mathrm{yr}$ & & & - & & & {$[0.031]$} \\
\hline No/don't know & - & - & - & 1.00 & - & - \\
\hline Yes & - & - & - & 2.22 & $(1.08-4.60)$ & 0.031 \\
\hline
\end{tabular}


times as likely to report high levels of interest in genetic testing than those who had not had a test.

\section{Models assessing associations between psychosocial variables and interest in genetic testing in unaffected men}

The unadjusted and adjusted models for prostate cancer risk perceptions and prostate cancer worry for unaffected men are presented in Table 4. Both worry and risk perceptions were significantly associated with interest in prostate cancer genetic testing in the models adjusted for correlated responses within families and family history. Those unaffected men who perceived their risk of getting prostate cancer to be higher had 2.05 (95\% CI: 1.34-3.13) times the odds of reporting high levels of interest in receiving a prostate cancer genetic test. For cancer worry, those with occasional worry had 1.82 (95\% CI: $1.18-$ 2.83) times the odds of reporting higher levels of interest in receiving a prostate cancer genetic test when compared with those who had no or little worry. In the adjusted model, however, only cancer worry remained significant. The odds of those with occasional worry were 1.74 (95\% CI: 1.07-2.83) times the odds of those with no or little worry in reporting high levels of interest in prostate cancer genetic testing.

\section{DISCUSSION}

The main objective of this study was to identify sociodemographic, psychosocial, medical, and health-related behavioral characteristics correlated with interest in genetic testing for prostate cancer risk in affected and unaffected men from HPC families. Overall, similar numbers of affected and unaffected family members (45 and 43\%, respectively) reported that they were "definitely interested" in participating in genetic testing for prostate cancer. These percentages are lower than those reported in the majority of previous studies of interest in prostate cancer genetic testing in unaffected men (between 74 and $98 \%)^{23,24,26,27,35}$ However, one study by Diefenbach et al. ${ }^{28}$ reported that only $24 \%$ of men with and without a family history would obtain prostate cancer genetic testing. To date, there are no previous studies of interest in genetic testing among affected men. Discrepancies between our findings and those of previous studies may be due to differences in how interest was measured.
For example, in this study, we dichotomized the variable into those who were definitely interested versus all other responses, whereas in several previous studies the variable was dichotomized into definitely or probably interested versus less interested. Discrepancies may also be due to the fact that there were differences in population demographics between our study and several of the other studies. For example, the men in our study tended to be older than men surveyed in previous studies of interest in prostate cancer genetic testing.

The high rate of interest in genetic testing in both affected and unaffected men in our sample is consistent with the rates reported for interest in both breast and colorectal cancer. ${ }^{13,17,19,36}$ Although there are several studies illustrating that men report less interest and uptake in genetic testing than women, ${ }^{13,19,37}$ a growing number of studies indicate that rates of interest are similarly high between men and women. ${ }^{14,38} \mathrm{~A}$ recent study by Peters et al. ${ }^{18}$ reported that a majority of both men and women reported interest in testicular cancer genetic testing. Two studies also indicated that men and women were equally interested in HNPCC testing, ${ }^{14,38}$ though there is also evidence indicating lower rates of interest in men. ${ }^{13,37}$ It is clear that the story with respect to gender and interest in genetic testing is still unfolding. As we develop genetic tests for male-specific cancers, elucidating men's preferences, motivations, and interest in genetic testing is of paramount concern. A recent survey of men's interest in genetic services for prostate cancer indicated that the majority of men had insufficient information about their genetic risk, and that they had very specific needs with respect to how they would like to receive genetic information. ${ }^{39}$ Taken together, these results suggest that future research should focus on the role of gender in the decision-making process to determine better methods for delivering test results.

To date there are only two studies in the literature that have examined interest in prostate cancer susceptibility genetic testing in men from HPC families. ${ }^{29,30}$ Both studies were conducted in unaffected relatives. Weinrich et al. ${ }^{30}$ examined sociodemographic correlates of interest in genetic testing and found that marital status was the only significant predictor of interest in testing. In our data, age and familiarity with genetic testing were strongly associated (age being negatively correlated and familiarity being positively correlated) with interest in prostate cancer genetic testing among affected and unaffected men. Several

Table 4 Prostate cancer risk perception and worry among unaffected HPC family members: Odds ratio (OR) and 95\% confidence interval $(\mathrm{Cl})$ estimates for interest in genetic testing for prostate cancer ${ }^{a, b}$

\begin{tabular}{|c|c|c|c|c|c|c|}
\hline \multirow[b]{2}{*}{ Characteristics } & \multicolumn{3}{|c|}{ Single-predictor models ${ }^{b}$} & \multicolumn{3}{|c|}{ Multivariable model ${ }^{c}$} \\
\hline & OR & $95 \% \mathrm{CI}$ & $P$ & OR & $95 \% \mathrm{CI}$ & $P$ \\
\hline \multicolumn{7}{|c|}{ Prostate cancer risk perception (scale score) } \\
\hline Lower personal risk & 1.00 & - & - & 1.00 & - & - \\
\hline Higher personal risk & 2.05 & $(1.34-3.13)$ & 0.001 & 1.48 & $(0.91-2.41)$ & 0.110 \\
\hline \multicolumn{7}{|c|}{ Cancer worry (scale score) } \\
\hline No/little worry & 1.00 & - & - & 1.00 & - & - \\
\hline Occasional worry & 1.82 & $(1.18-2.83)$ & 0.007 & 1.74 & $(1.07-2.83)$ & 0.027 \\
\hline
\end{tabular}


other studies of interest in genetic testing for colorectal cancer and breast cancer have shown similar associations between age and interest. ${ }^{14,16,40,41}$ Although familiarity with genetic testing is not the same as knowledge (e.g., an individual could be very familiar with the fact that genetic testing exists and still not understand how the test works), there is likely to be overlap between the two variables (i.e., it is likely that those who are not knowledgeable about genetics are also likely not to be familiar with genetics). Similar to our results on familiarity, several studies have shown a strong association between knowledge and interest in breast/ovarian and colorectal cancer genetic testing. ${ }^{14,16,40}$ Though it is important to note that a few studies have not found an association between age or knowledge and interest in genetic testing. ${ }^{17,42}$ The association between age, familiarity or knowledge, and interest in genetic testing across numerous types of cancer genetic susceptibility tests points to the importance of accounting for these factors when tailoring educational materials.

Among affected men, race/ethnicity remained a strong predictor of interest in genetic testing even after controlling for other significant predictors. The fact that minority groups were significantly less interested $(\mathrm{OR}=0.25 ; 95 \% \mathrm{CI}$ : $0.12-0.54)$ than whites in prostate cancer genetic testing points to the need for additional research to uncover why this discrepancy exists. There is evidence from the literature on breast/ovarian and colorectal cancer genetic testing that exposure to information about genetic testing is limited among African-Americans and concerns about the potential abuses of the information are higher than in whites. ${ }^{40,43-45}$ Thus, additional research is needed to evaluate the acceptability of genetic testing for prostate cancer in different racial/ethnic communities.

A higher percentage of affected men under the age of 65 were interested in genetic testing when compared with unaffected men ( 65 vs. $46 \%$ ). One possible explanation is that this might be related to the fact that older men might have had more affected sons and so believed that they had a hereditary form of the disease without the benefit of testing. This is consistent with at least one finding from the literature that showed that men with a familial risk for prostate cancer were concerned about the risk for their sons. ${ }^{26}$ This is also consistent with trends in breast cancer genetic testing, where one of the key motivators for obtaining testing is to pass mutation status information onto children. ${ }^{46}$ It is possible that interest in prostate cancer genetic testing in younger men was motivated by a desire to be able to tell as yet unaffected sons about the father's mutation status.

Finally, similar to other studies investigating genetic testing for prostate cancer, ${ }^{26,28}$ colorectal cancer, ${ }^{13,14}$ and breast/ovarian cancer, ${ }^{42,47}$ cancer worry and cancer risk perceptions were significantly associated with interest in genetic testing. Although risk perceptions and worry about prostate cancer were both significantly and positively associated with interest, only worry remained significant after adjusting for other predictors. These findings are consistent with one study of sons of prostate cancer cases ${ }^{26}$ and contrary to another study that found no association between perceived risk and interest in prostate cancer genetic testing. ${ }^{28}$ Placed into the larger context of genetic testing, these findings add to the growing literature on cancer risk and worry as strong predictors of interest in genetic testing and a variety of other health behaviors including prostate cancer screening. ${ }^{48}$ These results coupled with the fact that $60 \%$ of men in a recent study reported having insufficient information about their prostate cancer risk, ${ }^{39}$ indicate that men may respond to risk counseling and may also represent an underserved group that should be targeted for cancer risk counseling.

This study has several strengths and limitations to be considered when interpreting results. The study represents the largest investi- gation of HPC families reported to date, in which interest in genetic testing for prostate cancer susceptibility is examined in both affected and unaffected men. As noted previously, this is of particular importance because of the nature of the testing process and the importance of involving the proband and unaffected family members. Additionally, this study includes health-related behaviors, medical history characteristics, and characteristics of the prostate cancer diagnosis that have not been previously examined in relation to interest in genetic testing for prostate cancer susceptibility. Understanding these correlates and similarities and differences between affected and unaffected men with respect to genetic testing interest provides direction for creating educational programs and guiding clinical practice and recommendations.

This study also has some limitations. First, we asked individuals to answer about their level of interest in a hypothetical genetic test. The fact that a genetic test for prostate cancer does not currently exist means that test parameters, costs, and benefits are unknown, which leaves the respondent to speculate about each of these conditions. Thus, it may make it more difficult for the respondent to provide a truly informed answer. Furthermore, until a test is made available, it is impossible to know how well intentions are associated with actual genetic testing uptake. Several studies have reported a mismatch between intention and uptake with people tending to greatly overestimate their actual uptake. ${ }^{18,38}$ However, the rapid pace of genetic research necessitates the exploration of these issues before market release to prepare clinicians and patients for the influx of new genetic tests on the horizon. Second, the study sample is HPC family members enrolled in a project to identify prostate cancer susceptibility genes. Thus, this sample is likely to be highly interested in genetics and potentially more knowledgeable than other members of HPC families or the general population. Therefore, external validity may be an issue. External validity may also be an issue due to the fact that the sample contains only $10 \%$ non-whites, which is less than the actual percentage of nonwhites affected with prostate cancer (i.e., 16\%). This underrepresentation is somewhat common in genetics research because of historical governmental abuses of African-Americans and other racial/ethnic groups in medical research. Therefore, it should be noted as a potential source of bias and limitation in generalizing results across racial/ethnic minorities. Finally, a significant span of time lapsed between baseline and follow-up survey administration (mean $=6.1$ year). Although the time-sensitive variables such as screening behavior, health-related behaviors, and risk perceptions were assessed at the same time as the outcome variable, several demographic variables were only assessed at baseline. Although unlikely, it is possible that a few of the demographic variables such as marital or educational status could have changed between baseline and follow-up.

The emerging prostate cancer genetic susceptibility tests are not likely to follow the $B R C A 1 / 2$ model of a genetic test. Tests will include information about multiple genotypes conferring various risk information, and interpreting those results will require a high degree of skill in understanding probabilistic information. Overall, prostate cancer genetic testing results are likely to be more difficult for providers to interpret, patients to understand, and for both providers and patients to determine an appropriate plan for screening or treatment. This study has illuminated several predictors of interest in prostate cancer genetic testing among men from HPC families that should be considered when developing future educational materials for patients. We have also identified subgroups that may benefit most from targeted educational and psychosocial interventions and areas to consider exploring to identify individual motivations for prostate cancer genetic testing. 


\section{Human subjects}

This study was approved by the Institutional Review Board at Fred Hutchinson Cancer Research Center and informed consent was obtained from all participants.

\section{ACKNOWLEDGMENTS}

This work was supported by Biobehavioral Cancer Prevention and Control Training Grant R25 CA092408 (JNH), Pacific Northwest Prostate Cancer SPORE Career Development Program Fellowship P50 CA097186 (LMF), and RO1 CA080122 from the National Cancer Institute, with additional support from the Fred Hutchinson Cancer Research Center and the Intramural Program of the National Human Genome Research Institute (EAO). Julie N Harris is a Robert Wood Johnson Foundation Health \& Society Scholar at the University of California in San Francisco and Berkeley, CA. They also thank all of the individuals who participated in the PROGRESS study.

\section{REFERENCES}

1. Jemal A, Siegel R, Ward E, et al. Cancer statistics, 2008. CA Cancer J Clin 2008;58:71-96.

2. Bruner DW, Moore D, Parlanti A, Dorgan J, Engstrom P. Relative risk of prostate cancer for men with affected relatives: systematic review and meta-analysis. Int J Cancer 2003;107:797-803.

3. Zeegers MP, Jellema A, Ostrer H. Empiric risk of prostate carcinoma for relatives of patients with prostate carcinoma: a meta-analysis. Cancer 2003; 97:1894-1903.

4. Langeberg WJ, Isaacs WB, Stanford JL. Genetic etiology of hereditary prostate cancer. Front Biosci 2007;12:4101-4110.

5. Amundadottir LT, Sulem P, Gudmundsson J, et al. A common variant associated with prostate cancer in European and African populations. Nat Genet 2006;38:652-658.

6. Gudmundsson J, Sulem P, Manolescu A, et al. Genome-wide association study identifies a second prostate cancer susceptibility variant at 8q24. Nat Genet 2007;39:631-637.

7. Gudmundsson J, Sulem P, Steinthorsdottir V, et al. Two variants on chromosome 17 confer prostate cancer risk, and the one in TCF2 protects against type 2 diabetes. Nat Genet 2007;39:977-983.

8. Zheng S, Sun J, Wiklund F, et al. Cumulative association of five genetic variants with prostate cancer. N Engl J Med 2008;358:910-919.

9. Bratt O. Hereditary prostate cancer: clinical aspects. J Urol 2002;168:906-913.

10. Steinberg G, Carter B, Beaty T, Childs B, Walsh P. Family history and the risk of prostate cancer. Prostate 1990;17:337-347.

11. Carter BS, Bova GS, Beaty TH, et al. Hereditary prostate cancer: epidemiologic and clinical features. J Urol 1993;150:797-802.

12. Kopper L, Timar J. Genomics of prostate cancer: is there anything to "translate"? Pathol Oncol Res 2005;11:197-203.

13. Petersen G, Larkin E, Codori A, et al. Attitudes toward colon cancer gene testing: survey of relatives of colon cancer patients. Cancer Epidemiol Biomarkers Prev 1999;8(4 Part 2):337-344.

14. Glanz K, Grove J, Lerman C, Gotay C, LeMarchand L. Correlates of intentions to obtain genetic counseling and colorectal cancer gene testing among at-risk relatives from three ethnic groups. Cancer Epidemiol Biomarkers Prev 1999;8(4 Part 2):329-336.

15. Lerman C, Marshall J, Audrain J, Gomez-Caminero A. Genetic testing for colon cancer susceptibility: anticipated reactions of patients and challenges to providers. Int J Cancer 1996;69:58-61.

16. Botoroff J, Ratner P, Balneaves L, et al. Women's interest in genetic testing for breast cancer risk: the influence of sociodemographics and knowledge. Cancer Epidemiol Biomarkers Prev 2002;11:89-95.

17. Kinney A, Choi Y-A, DeVellis B, Kobetz E, Millikan R, Sandler R. Interest in genetic testing among first-degree relatives of colorectal cancer patients. Am J Prev Med 2000;18:249-252.

18. Peters N, Domchek S, Rose A, Polis R, Stopfer J, Armstrong K. Knowledge, attitudes, and utilization of BRCA1/2 testing among women with early-onset breast cancer. Genet Test 2005;9:48-53.

19. Lerman $\mathrm{C}$, Narod $\mathrm{S}$, Sculman $\mathrm{K}$, et al. BRCA1 testing in families with hereditary breast-ovarian cancer: a prospective study of patient decision making and outcomes. JAMA 1996;275:1885-1892.

20. Peters J, Vadaparampil S, Kramer J, et al. Familial testicular cancer: interes in genetic testing among high-risk family members. Genet Med 2006;8:760770 .

21. d'Agincourt-Canning L, Baird P. Genetic testing for hereditary cancers: the impact of gender on interest, uptake, and ethical considerations. Crit Rev Oncol/Hematol 2006;58:114-123.

22. Doukas DJ, Fetters MD, Coyne JC, McCullough LB. How men view genetic testing for prostate cancer risk: findings from focus groups. Clin Genet 2000;58:169-176

23. Li Y, Doukas DJ. Health motivation and emotional vigilance in genetic testing for prostate cancer risk. Clin Genet 2004;66:512-516.

24. Doukas DJ, Li Y. Men's values-based factors on prostate cancer risk genetic testing: a telephone survey. BMC Med Genet 2004;5:28.

25. Doukas DJ, Localio AR, Li Y. Attitudes and beliefs concerning prostate cancer genetic screening. Clin Genet 2004;66:445-451.

26. Bratt O, Kristoffersson U, Lundgren R, Olsson H. Sons of men with prostate cancer: their attitudes regarding possible inheritance of prostate cancer, screening, and genetic testing. Urology 1997;50:360-365.

27. Cormier L, Valeri A, Azzouzi R, et al. Worry and attitude of men in at-risk families for prostate cancer about genetic susceptibility and genetic testing. Prostate 2002;51:276-285.

28. Diefenbach M, Schnoll R, Miller S, Brower L. Genetic testing for prostate cancer. Willingness and predictors of interest Cancer Pract 2000;8:82-86.

29. Bratt O, Damber J, Emanuelsson M, et al. Risk perception, screening practice, and interest in genetic testing among unaffected men in families with hereditary prostate cancer. Eur J Cancer 2000;36:235-241.

30. Weinrich S, Royal C, Pettaway C, et al. Interest in genetic prostate cance susceptibility testing among African American men. Cancer Nurs 2002;25: 28-34.

31. Stanford J, McDonnell S, Friedrichsen D, et al. Prostate cancer and genetic susceptibility: a genome scan incorporating disease aggressiveness. Prostate 2006; 66:317-325.

32. Lerman C, Trock B, Rimer B, Jepson C, Brody D, Boyce A. Psychological side effects of breast cancer screening. Health Psychol 1991;10:259-267.

33. Bowen DJ, Ludman E, Press N, Vu T, Burke W. Achieving utility with family history: colorectal cancer risk. Am J Prev Med 2003;24:177-182.

34. Ries L, Melbert D, Krapcho M, et al. SEER Cancer Statistics Review, 1975-2004. Bethesda, MD, 2007. Based on November 2006 data submission, posted to the SEER website.

35. Miesfeldt S, Jones S, Cohn W, et al. Men's attitudes regarding genetic testing for hereditary prostate cancer risk. Adult Urol 2000;55:46-50.

36. Tambor E, Rimer B, Strigo T. Genetic testing for breast cancer susceptibility: awareness and interest among women in the general population. Am J Med Genet 1997;68:43-49.

37. Wagner A, Tops C, Wingen J, et al. Genetic testing in hereditary nonpolyposis colorectal cancer families with a MSH2, MLH1, OR MSH6 mutation. $J$ Med Genet 2002;163:573-582.

38. Hadley D, Jenkins J, Diamond E, et al. Genetic counseling and testing in families with hereditary nonpolyposis colorectal cancer. Arch Intern Med 2003; $163: 573-582$

39. Gaff CL, Cowan R, Meiser B, Lindeman G. Genetic services for men: the preferences of men with a family history of prostate cancer. Genet Med 2006;8:771-778.

40. Satia J, McRitchie S, Kupper L, Halbert CH. Genetic testing for colon cancer among African-Americans in North Carolina. Prev Med 2006;42:51-59.

41. Kinney A, Choi Y-A, DeVellis B, Millikan R, Kobetz E, Sandler R. Attitudes toward genetic testing in patients with colorectal cancer. Cancer Pract 2000;8:178-186.

42. Capelli M, Surh L, Humphreys L, et al. Psychological and social determinants of women's decisions to undergo genetic counseling and testing for breast cancer. Clin Genet 1999;55:419-430.

43. Thompson H, Valdimarsdottir H, Jandorf L, Redd W. Perceived disadvantages and concerns about abuses of genetic testing for cancer risk: differences across African-American, Latina, and Caucasian women. Patient Educ Couns 2003;51:217-227.

44. Hughes C, Gomez-Caminero A, Benkendorf J, et al. Ethnic differences in knowledge and attitudes about BRCA1 testing in women at increased risk. Patient Educ Couns 1997;32:51-62.

45. Hughes C, Lerman C, Lustbader E. Ethnic differences in risk perception among women at increased risk for breast cancer. Breast Cancer Res Treat 1996;40:25-35.

46. Lerman C, Daly M, Masny A, Balshem A. Attitudes about genetic testing for breast-ovarian cancer susceptibility. J Clin Oncol 1994;12:843-850.

47. Lipkus I, Iden D, Terrenoire J, Feaganes J. Relationships among breast cancer concern, risk perceptions, and interest in genetic testing for breast cancer susceptibility among African-American women with and without a family history of breast cancer. Cancer Epidemiol Biomarkers Prev 1999; 8:533-539.

48. Bloom J, Stewart S, Oakley-Girvans I, Banks P, Chang S. Family history, perceived risk, and prostate cancer screening among African American men. Cancer Epidemiol Biomarkers Prev 2006;15:2167-2173. 